\title{
HIV COUNSELLING
}

\author{
Linda-Gail Bekker, $M B C h B, F C P, P h D$ \\ Infectious Disease Clinical Research Unit, UCT Lung Institute, Cape Town
}

The HIV epidemic more than any other has brought with it the need for health care workers to have counselling skills. The life-changing nature of an HIV diagnosis requires that all persons are fully informed of the consequences of a positive result before they are tested (pretest counselling) and thereafter be told the result and given support if it is positive (post-test counselling). There are a number of other situations in the context of HIV where counselling is required, e.g. bereaved family members, loss of reproductive capability, etc. It is therefore in the best interests of the health care professional not only to know the resources to call upon for more advanced counselling support but also to develop some personal basic counselling skills.

Counselling in a health care setting consists of a supportive relationship in a structured environment where one person helps another person to:

Know more about a situation/illness/diagnosis.

- Contain his/her emotions.

Explore and define his/her feelings, thoughts and actions around the diagnosis.

Find solutions to problems that he/she will encounter.

Develop coping skills for the future.

HIV counselling aims to:

- Provide a supportive environment.

Help clients manage their problems and issues.

Explore coping skills they have used before and develop new ones.

-1. Empower clients to become self-sufficient in dealing with emerging issues/problems.

an Counsel HIV-negative clients so that they know how to remain negative.

- Counsel HIV-positive clients on how to avoid crossinfection and how to prevent infecting others.

Explore options with the client that will help them to bring about necessary changes in behaviour. These

This article has been adapted by Linda-Gail Bekker from a chapter by Carroll Jacobs in the Handbook of HIV Medicine (reviewed elsewhere in this issue). It is recommended that the full chapter in the handbook be referred to. options should include abstinence, monogamy (mutual faithfulness) and the correct use of condoms.

Probably the most common reason for health professionals to counsel South Africans today is in the context of HIV testing.

The Health Professions Council of South Africa and the South African Medical Association recommend that since an HIV test interferes with a person's rights to freedom and security of person and privacy, a person may only be tested

athis/her own request

after $s /$ he has given informed consent, preferably written

- he/she may otherwise be tested when authorised by legislation or a court order.

Before testing, the health professional should:

- Check for legislative processes that may regulate HIV testing, e.g. prisons, schools, workplace, insurance policies, etc.

- Confirm that there is a clinical indication.

Ensure that pretest counselling has been done adequately, or do so personally.

Ensure that the client has been fully informed and thereafter consented.

즐 $\mathrm{Be}$ in a position to ensure confidentiality.

In the absence of a test, emergency treatment may not be withheld. In certain well-defined high-risk or exposureprone procedures, the patient should be informed and asked to consent. If the patient declines, s/he should be managed as if positive.

Testing as part of research is permitted only with tne candidate's free and informed consent. Testing an existing blood sample can only be done if the patient consents.

Unlinked and anonymous testing is permitted by the Department of Health if done by a national or local health authority. Proper ethical guidelines should be adhered to and there should be security safeguards for confidentiality.

A general poster in the ward or clinic room that 'all patients will be tested for HIV' does NOT constitute informed consent. Nor should a patient just be presented with a leaflet or referred to another organisation for information. 


\section{PRETEST COUNSELLING}

Checklist for pretest counselling:

Assure the client that both counselling and testing are confidential procedures.

- Be sure that if more than one session is required it can be offered.

-1 Provide information about HIV infection and transmission and its links to AIDS, sexually transmitted infections and tuberculosis.

- Provide information on the technical aspects of testing, the 'window period' and its implications and the meaning of the terms 'positive' and 'negative'.

- Discuss the implications of a positive or negative diagnosis.

- Provide information about the client's legal rights in terms of who to tell (sexual partner/s) or not to tell (e.g. the employer, third parties, etc.). Clients are not obliged to tell anyone apart from their sexual partners.

질 Evaluate risk behaviour: find out why the individual wants to be tested, and the nature and extent of previous and present high-risk behaviour, and discuss the steps that they should take to prevent future infection or transmission.

Determine the client's coping resources and support systems in the event of a positive result.

- Contain the client's emotions as they deal with issues about relationships.

- Determine whether the client wishes to be tested that day or not, and whether they would like to receive the result that day or not.

Assure the client that you respect their decision.

Provide a sense of support and hope for the client.

A person who has tested HIV-positive may never have the same quality of life again. HIV-positive people who are properly and appropriately counselled not only feel better following the support, but also are better able to talk about their fears and feelings, and to plan their future. With ongoing emotional and psychological support the HIVpositive person can change his or her behaviour from destructive living to positive living.

Both pre- and post-test counselling are very important. It is dangerous to compromise the counselling process and take short cuts in the counselling room.

\section{POST-TEST COUNSELLING}

Post-test counselling helps the client to work through the crisis and other issues that may arise as a result of being told their HIV status.

Giving the results.

Give the results as soon as they are received. Do not prolong the suspense with inane conversation.

- Give the results face to face, never telephonically and never through third parties.

-1 The person who conducted the pretest counselling should wherever possible also give the result and posttest counselling.

- Let the client choose whether they want the result that day or not. People may need more time to consider the impact of a possible HIV-positive result on their lives before they receive the result.

- If the result is positive, do not give it unless you or a back-up support can see the client the following day.

If the HIV result is negative:

Discuss the window period.

aeinforce the message of prevention and safer sexual practices.

- Pick up on any other issues that may have been raised in the pretest counselling session.

Discuss referral if necessary, especially if there are ongoing risk factors.

HIV positive result:

Initially:

- Concentrate on managing the resultant crisis and addressing the client's immediate concerns.

- Be careful of information overload in the first encounters.

Later loften at a follow-up appointment the next day, if possible)

- Continue to contain the client's emotions.

- Answer any questions.

- Remind the client of the need for partner notification though they may still need some time and support for this.

Introduce concepts such as:

a the need for medical assessment and where to get it

- safer sex

- the cost and availability of antiretroviral therapies (ART) and drug trials

- the importance of a well-balanced diet, rest and exercise

1. the need to eliminate alcohol, smoking or drugs.

Ongoing counselling helps the client to deal with issues such as partner notification, relationship difficulties queries about health and treatment and disclosure to others. With written permission, the counsellor may liaise with other caregivers. Develop a good working relationship with them, as you may be able to share contacts and referral sources. 